\title{
Are bipolar II patients cognitively impaired? A systematic review
}

\author{
B. Solé ${ }^{1}$, A. Martínez-Arán ${ }^{1}$, C. Torrent ${ }^{1}$, C. M. Bonnin ${ }^{1}$, M. Reinares $^{1}$, D. Popovic ${ }^{1,2}$, \\ J. Sánchez-Moreno ${ }^{1}$ and E. Vieta ${ }^{1 *}$ \\ ${ }^{1}$ Bipolar Disorders Program, Hospital Clinic, University of Barcelona, IDIBAPS, CIBERSAM, Barcelona, Spain \\ ${ }^{2}$ Department of Psychiatry, Neurobiology, Pharmacology and Biotechnology, University of Pisa, Pisa, Italy
}

Background. There is evidence that bipolar disorder (BD) is associated with significant neurocognitive deficits and this occurs in individuals with BD type I (BD I) and with BD type II (BD II). Only a few studies have focused on cognitive impairment in BD II. The aim of this study was to describe the pattern of cognitive impairment in patients with BD II, in order to identify specific cognitive deficits that distinguish BD II from BD I patients as well as from healthy subjects.

Method. We performed a systematic review of the literature of neuropsychological studies of BD II published between 1980 and July 2009. Fourteen articles fulfilled the inclusion criteria and were included in this review.

Results. Main cognitive deficits found in BD II include working memory and some measures of executive functions (inhibitory control) and approximately half of the studies also detected verbal memory impairment.

Conclusions. There are subtle differences between the two subtypes regarding cognition. This may suggest neurobiological differences between the two subgroups which will be helpful in order to determine cognitive endophenotypes in BD subtypes.

Received 23 June 2010; Revised 7 November 2010; Accepted 22 December 2010; First published online 28 January 2011

Key words: Bipolar disorder II, cognition, neuropsychology, systematic reviews.

\section{Introduction}

Bipolar disorder (BD) occurs in multiple forms and degrees of severity. Whereas the Diagnostic and Statistical Manual of Mental Disorders (DSM-IV) includes the BD II subtype, the International Classification of Diseases, 10th Revision (ICD-10) does not recognize it as a specified nosological category (Vieta \& Suppes, 2008).

The clinical course of BD II is characterized by the presence or history of one or more major depressive episodes and at least one hypomanic episode lasting at least 4 days (APA, 2000). It is often initially misdiagnosed as unipolar depression, adjustment disorder or personality disorder (Berk \& Dodd, 2005). Many clinicians still consider BD II as a mild form of classical BD, although the data indicate that it may be associated with high morbidity and mortality, including higher episode frequency, co-morbidity, suicidal behaviour and rapid cycling as compared with BD I

* Address for correspondence: Dr E. Vieta, Bipolar Disorders Program, Clinical Institute of Neuroscience, University Clinic Hospital of Barcelona, CIBERSAM, Villarroel 170, 08036-Barcelona, Spain.

(Email: evieta@clinic.ub.es)
(Vieta et al. 1997, 1999). Misdiagnosing BD II may also affect therapeutic decisions and therefore the course and prognosis of bipolar patients (Vieta \& Suppes, 2008). An early age at onset of BD II has been associated with a higher degree of severity, a poorer treatment response and a worse prognosis (Engstrom et al. 2003).

The estimated prevalence of BD II is from $0.5 \%$ to $6 \%$ depending on the flexibility of the application of diagnostic criteria (Akiskal, 1996; Angst, 1998; Benazzi, 1999).

In the past, several reviews have been published supporting recognition of BD II as a distinct category within mood disorders (Vieta \& Suppes, 2008).

Emerging evidence suggests considering cognitive impairment as a trait marker in BD, this impairment being present even during euthymic periods. Recently published meta-analyses have shown that executive functions and verbal memory are the most impaired cognitive domains in euthymic patients (Robinson et al. 2006; Torres et al. 2007) as well as attention/ processing speed (Torres et al. 2007). These neurocognitive deficits have been postulated to constitute trait markers or endophenotypes of BD (Glahn et al. 2004; Savitz et al. 2005), but the specific neurocognitive and 
therefore endophenotypic status of BD II and other bipolar spectrum disorders remains unclear (Savitz et al. 2008). Moreover, the concept of allostatic load is interesting in order to explain cognitive impairment in BD. The increased allostatic load in bipolar patients may be related to the cognitive decline seen among such patients as well as other pathophysiological mechanisms involved in illness progression. The main mediators of allostatic load are the dysregulation of the hypothalamic-pituitary-adrenal axis, and altered immunity, pro-inflammatory and oxidative stress states (Kapczinski et al. 2008).

Only a few studies have focused on cognitive impairment in BD II, probably due to the fact that BD II is an underdiagnosed entity. Most data regarding neurocognition in BD come from studies focusing on BD I patients or mixed samples of BD I and BD II subjects.

In one of the first studies comparing BD I and BD II, euthymic BD II patients had significantly less insight and higher level of subjective cognitive complaints than BD I patients (Pallanti et al. 1999). It is noteworthy that cognitive impairment, particularly memory deficits, may have negative implications in the functional outcome of bipolar patients (Martínez-Arán et al. $2004 a, b)$. Between $30 \%$ and $50 \%$ of patients with BD experience significant social disability that may be related to persistent cognitive impairment (Zarate et al. 2000) and there is a paucity of studies focusing on this topic in BD II.

The main aim of this critical review is to examine the existing literature on cognitive impairment in BD II in order to identify specific cognitive deficits that distinguish BD II from BD I patients, as well as from healthy subjects, therefore having implications for the patho-aetiology and nosology of this disorder.

\section{Methodology}

A comprehensive PubMed/Medline search was carried out in order to conduct an objective review of the available literature on the neuropsychology of BD II. The search was supplemented by manually reviewing reference lists from the identified publications. Only English-language articles published from 1980 to July 2009 were included in the present paper, using the search term 'bipolar II' cross-referenced with 'cognition', 'cognitive function', 'cognitive impairment', 'neuropsychological', 'neuropsychological function', 'neurocognitive', 'attention', 'memory', 'executive function' and 'intellectual function'. Eligibility criteria were: (a) studies that included a comparison group [psychiatric or healthy control (HC) group] or normative data for standardized tests; $(b)$ groups formed of more than 10 BD II subjects; (c) published between 1980 and July 2009; (d) adult patients (aged 18-65 years); (e) the use of standardized diagnostic criteria, such as DSM; $(f)$ selection of standardized or wellestablished cognitive tasks; and $(g)$ provided clear descriptive and comparative statistics of cognitive function measures.

\section{Results}

The systematic search yielded 55 articles, out of which 15 fulfilled the inclusion criteria. Table 1 summarizes the findings of the studies included in this review. Results will be shown according to different neurocognitive domains.

\section{General intellectual function}

One of the selected studies did not assess pre-morbid intelligence quotient (IQ) or current IQ (Hsiao et al. 2009). Most of the other studies did not find significant differences between BD I, BD II and the HC group in the estimated current IQ, using different subtests of the Wechsler Adult Intelligence Scale or the Wechsler Abbreviated Scale of Intelligence (WASI), or the premorbid IQ using the National Adult Reading Test (NART) (Torrent et al. 2006; Taylor Tavares et al. 2007; Andersson et al. 2008; Holmes et al. 2008; Savitz et al. 2008). Harkavy-Friedman et al. (2006) did not find significant differences in estimated current IQ assessed by the Peabody Picture Vocabulary either. It is noteworthy that Simonsen et al. (2008) did not find any difference between the three groups (BD I, BD II and $\mathrm{HC}$ ) in pre-morbid IQ assessed by the NART; however, current IQ assessed by the WASI revealed that the mean score of BD I patients was lower than that of the HC group, whilst BD II patients did not differ significantly from either group. However, although these differences were statistically significant, they were not significant from a clinical point of view.

Only one study found that both BD I and BD II patient groups differed significantly from the $\mathrm{HC}$ group as to pre-morbid IQ, but they did not differ significantly one from another (Dittmann et al. 2008). The baseline differences in pre-morbid IQ among groups might have affected the results on neuropsychological performance.

Furthermore, only one study considered an index of IQ change in order to assess intellectual decline and found that BD II patients scored significantly lower than BD I on full-scale IQ change (Summers et al. 2006). Bruno et al. (2006), using the same sample of BD patients, aimed to correlate cognitive performance (IQ change) with structural brain abnormalities using neuroimaging techniques. An association between fronto-temporal abnormalities and decline in IQ in BD was detected as well as that the structural/cognitive 
Table 1. Studies on neurocognition including patients with BD II

\begin{tabular}{|c|c|c|c|c|}
\hline Authors & Sample & Cognitive measures & Results & Comments \\
\hline Berns et al. (2002) & $13 \mathrm{BD} \mathrm{II} v .14 \mathrm{HC}$ & RT task (novel spatial-motor sequence) & $\begin{array}{l}\text { BD II }=\text { HC in RT } \\
\text { Differences in brain activation, BD II } \\
\text { reacted biologically in a way } \\
\text { congruent with affective lability }\end{array}$ & $\begin{array}{l}\text { Lack of parietal activation in patients; } \\
\text { widespread medial prefrontal and limbic } \\
\text { activation }\end{array}$ \\
\hline $\begin{array}{l}\text { Martínez-Aran } \\
\text { et al. }(2004 a)\end{array}$ & $\begin{array}{l}108 \mathrm{BD}(30 \mathrm{D} v \\
34 \mathrm{M} v .44 \mathrm{E}) v \\
30 \mathrm{HC}\end{array}$ & $\begin{array}{l}\text { WAIS Voc; WCST, SCWT, FAS, Animal Naming; } \\
\text { WAIS digits, TMT-A TMT-B; CVLT, WMS-R } \\
\text { Logical Memory, WMS-R Visual Reproduction }\end{array}$ & BD I < BD II: CVLT & $\begin{array}{l}\text { No differentiation in clinical status in the } \\
\text { analysis of diagnostic subtype }\end{array}$ \\
\hline Bruno et al. (2006) & $11 \mathrm{BD} \mathrm{II} v .25 \mathrm{BD} \mathrm{I}$ & $\begin{array}{l}\text { IQ NART; IQ WAIS-R; Recognition Memory Test, } \\
\text { PALT, ROCFT, Doors and People Test; MCST; } \\
\text { SWM, IDED of CANTAB }\end{array}$ & $\begin{array}{l}\text { Structural/cognitive correlates more } \\
\text { extensive in BD II than BD I }\end{array}$ & $\begin{array}{l}\text { Same sample as in Summers et al. (2006) } \\
\text { study. } \\
\text { Structural brain abnormalities were } \\
\text { correlated with decline in IQ. They also } \\
\text { explored differences between clinical } \\
\text { subtypes }\end{array}$ \\
\hline $\begin{array}{l}\text { Harkavy- } \\
\text { Friedman et al. } \\
(2006)\end{array}$ & $\begin{array}{l}19 \mathrm{BD} \mathrm{II} v .32 \mathrm{BD} \mathrm{I} \\
v .58 \mathrm{HC}\end{array}$ & $\begin{array}{l}\text { PPVT; Finger Tapping Test, Brief Computerized } \\
\text { RT; WAIS-III Digit Symbol, TMT-A, TMT-B; } \\
\text { Buschke Selective Reminding Test, BVRT; CPT, } \\
\text { Stroop; N Back Test, A not B RT; Go-No-Go, } \\
\text { Time Estimation Test; FAS }\end{array}$ & $\begin{array}{l}\text { BD I, BD II }<\text { HC: Digit Symbol, N Back } \\
\text { Test, Go-No-Go } \\
\text { BD II }<\text { HC: A not B RT, FAS } \\
\text { (BD I = BD II, HC) } \\
\text { BD II }<\text { HC, BD I: RT, Stroop }\end{array}$ & $\begin{array}{l}\text { Patients in a depressive episode with } \\
\text { suicide attempt }\end{array}$ \\
\hline $\begin{array}{l}\text { Summers } \\
\text { et al. (2006) }\end{array}$ & $11 \mathrm{BD}$ II $v .25 \mathrm{BD} \mathrm{I}$ & $\begin{array}{l}\text { IQ NART, IQ WAIS; Recognition Memory Test, } \\
\text { PALT, ROCFT, Doors and People Test; TMA-A; } \\
\text { Graded Naming Test; MCST, SCWT, COWAT, } \\
\text { Hayling Sentence Completion Test, CANTAB } \\
\text { SWM and IDED Set-Shift, TMT-B; Emotional } \\
\text { expression multimorph task }\end{array}$ & $\begin{array}{l}\text { BD I, BD II: impaired in recognition of } \\
\text { surprise } \\
\text { BD II < BD I: IQ change, memory } \\
\text { (RMF, PALT1, ROCFT) and executive } \\
\text { functions (TMT-B, SWM) } \\
\text { BD I: impaired in verbal recognition, } \\
\text { SWM } \\
\text { BD II: impaired verbal recognition, } \\
\text { SWM, recognition for faces, shape test, } \\
\text { ROCFT, PALT1, Heyling Sentence Test, } \\
\text { Stroop, IDED set-shift }\end{array}$ & $\begin{array}{l}\text { Nine patients had a current depressive } \\
\text { episode (no differences between subtypes } \\
\text { BD I } v \text {. BD II). No patients with current } \\
\text { manic/hypomanic episode. The effects of } \\
\text { depressive symptomatology were } \\
\text { controlled for }\end{array}$ \\
\hline $\begin{array}{l}\text { Torrent et al. } \\
\text { (2006) }\end{array}$ & $\begin{array}{l}33 \mathrm{BD} \mathrm{II} v .38 \mathrm{BD} \mathrm{I} \\
v .35 \mathrm{HC}\end{array}$ & $\begin{array}{l}\text { WAIS Voc; WCST, SCWT, FAS, Animal Naming; } \\
\text { WAIS Digits, TMT-A, TMT-B; CVLT }\end{array}$ & $\begin{array}{l}\text { BD I }<\text { BD II }<\text { HC: CVLT, SCWT } \\
\text { BD I, BD II }<\text { HC: attention (TMT-A, } \\
\text { digits forward), WM (digit backward) }\end{array}$ & $\begin{array}{l}\text { Euthymic patients during } 6 \text { months } \\
(\text { HAMD } \leqslant 8, \text { YMRS } \leqslant 6 \text { ) }\end{array}$ \\
\hline
\end{tabular}




\begin{tabular}{|c|c|c|}
\hline Authors & Sample & Cognitive measures \\
\hline $\begin{array}{l}\text { Taylor Tavares } \\
\text { et al. (2007) }\end{array}$ & $\begin{array}{l}17 \mathrm{BD} \mathrm{II} v .22 \\
\mathrm{MDD} v .25 \mathrm{HC}\end{array}$ & $\begin{array}{l}\text { WASI : CANTAB (PRM, SRM, SSP, SMTS \& } \\
\text { DMTS, SWM, IDED); CGT; IST }\end{array}$ \\
\hline $\begin{array}{l}\text { Andersson et al. } \\
(2008)\end{array}$ & $25 \mathrm{BD}$ II $v .28 \mathrm{HC}$ & $\begin{array}{l}\text { WASI Voc, Matrices; PASAT; SCWT, FAS; } \\
\text { CVLT-II; ROCFT; WAIS-R Digit Symbol ERP }\end{array}$ \\
\hline $\begin{array}{l}\text { Dittmann et al. } \\
\text { (2008) }\end{array}$ & $\begin{array}{l}38 \mathrm{BD} \mathrm{II} v .65 \mathrm{BD} \mathrm{I} \\
v .62 \mathrm{HC}\end{array}$ & $\begin{array}{l}\text { HAWIE-R Information; RBANS Form-A; TMT-A, } \\
\text { TMT-B; WAIS-III Letter-Number Sequencing }\end{array}$ \\
\hline
\end{tabular}

(2008)

$$
\text { v. } 62 \mathrm{HC}
$$

Holmes et al. (2008)

Savitz et al. (2008)

Simonsen et al. (2008)
33 Medicated BD (6 BD I, 27 BD II) v. 32

unmedicated BD (7 BD I, 25 BD II) v. $52 \mathrm{HC}$

19 BD II $v .49$ BD I v. 44 MDD-R $v$. 33 MDD-S $v .20$ other DSM-IV $v$. 65 unaffected

relatives

\section{BD II $v .42$ BD I} v. $124 \mathrm{HC}$
Results

Comments

SA-WAIS General Knowledge; Digits ; COWAT, WCST, SCWT; ROCFT; RAVLT

IQ NART, IQ WASI; WMS-III logical memory, CVLT-II ; WAIS-III Digits, WM-MA; D-KEFS Verbal Fluency; D-KEFS Colour-Word Interference Test
BD II $=\mathrm{HC}:$ on all neuropsychological measures

MDD < HC: SWM, IST

EDS (IDED)

MDD < BD II: CGT (quality of decision after a loss trial)

BD II $<$ HC: on all neuropsychological measures except FAS

BD II $>$ HC: MMN latency

BD I $<$ HC: on all domains except visual/constructional abilities BD II $<$ HC: psychomotor speed, WM, visual/constructional abilities and executive functions $\mathrm{BD}$ I= BD II ; on all domains

Medicated BD, particularly BD II: deficits on affective processing (positive) and sustained attention (RVP)

Medicated $\mathrm{BD}=$ unmedicated $\mathrm{BD}=\mathrm{HC}:$ PRM, SWM

BD I < unaffected relatives: RAVLT

$\mathrm{BD} \mathrm{II}=$ unaffected relatives

Unmedicated depressed patients during 3 weeks ( 8 weeks for fluoxetine)

Euthymic patients or middle to moderate depressed patients.

Authors assessed neurocognitive functioning combining neuropsychological test and ERP

Euthymic patients (at least 1 month). Results supported a similar neuropsychological profile in both subtypes

Patients tested during depressed mood states.

Additional tests omitting BD I patients to determine the impact of this smaller group on the overall results

Patients tested in a euthymic or at least relatively euthymic state (Beck Depression Inventory <10, ASRM <6).

Assessment of neuropsychological performance while controlling for childhood trauma, alcohol abuse and medication

BD I < BD II, HC: logical memory, CVLT

BD I<HC: digits backward, fluency set-shifting

BD I, BD II < HC: WM-MA, phonetic fluency, Colour-Word Interference Test manic patients

$24 \%$ BD I and $13 \%$ BD II had clinically significant cognitive impairment ( $\leqslant 1.5$ S.D.)
Excluded severely depressed or severely 
Simonsen et al.

$102 \mathrm{SZ}$ v. $27 \mathrm{SZA}$ v. $80 \mathrm{BD}$ I $v .56$

BD II $v .280 \mathrm{HC}$

NART-IQ; WMS-III Logical Memory, CVLT-II

WAIS-III Digit Symbol; Digits, WM-MA;

D-KEFS Verbal Fluency; D-KEFS Colour-Word Interference Test

Hsiao et al. (2009) 37 BD II $v .30$ BD I WMS-III (Logical Memory I and II; VPA I and II, v. $22 \mathrm{HC}$ Faces I and II, Family Pictures I and II, Digit Span
BD I $<$ HC $:$ SPM, VERT-K BD II $>$ HC: VIEMER-K

Diagnostic subtype only affects two verbal recall measures (WMS-III logical memory, CVLT-II)

\section{BD I < BD II, HC: WMS-III, TMT-B} Spatial Span); WAIS-III Digit Symbol, TMT-A; TMT-B
$\mathrm{BD} \mathrm{I}=\mathrm{BD} \mathrm{II}=\mathrm{HC}$ : Visual Memory, TMT-A

BD I, BD II $<$ HC: WM BD I $<$ BD II $<$ HC: Digit Symbol
Euthymic patients or with residual affective symptoms (MADRS $<18$ ). Two patients with YMRS $>8$

Authors investigated the role of lifetime history of psychosis for neurocognitive functioning. History of psychosis explained the neurocognitive variance in $\mathrm{BD}$ better than diagnostic subtype

Patients in an interepisode state (HAMD $\leqslant 7$ YMRS $\leqslant 6$ ) for at least 1 week before the assessment.

Using Memory Index for WMS-III.

Pre-morbid IQ was not estimated

ASRM, Altman Self-Rating Mania Scale; BD, bipolar disorder; BD I, bipolar disorder type I; BD II, bipolar disorder type II; BVRT, Benton Visual Retention Test; CANTAB, Cambridge Neuropsychological Test Automated Battery; CGT, Cambridge Gamble Test; COWAT, Controlled Oral Word Association Test; CPT, Continuous Performance Test; CVLT, California Verbal Learning Test; D, depressed patients; D-KEFS, Delis Kaplan Executive Functioning Scale; DSM, Diagnostic and Statistical Manual of Mental Disorders; E, euthymic patients; EDS, Extra-Dimensional Shift; ERP, event-related potentials; FAS, letter fluency test; HAWIE-R, German version of WAIS-R Information; HC, healthy controls; HAMD, Hamilton Depression Rating Scale; IDED, Intra-Dimensional Extra-Dimensional Set Shifting; IQ, intelligence quotient; IST, Information Sampling Test; M, manic or hypomanic patients ; MADRS, Montgomery Asberg Depression Rating Scale; MCST, Modified Wisconsin Card Sorting Test; MDD, major depressive disorder ; MDD-R, major depressive disorder - recurrent; MDD-S, major depressive disorder - single; MMN, mismatch negativity; NART, National Adult Reading Test; PALT, Paired Associates Learning Test (Warrington, 1996); PASAT, Paced Auditory Serial Addition Test; PPVT, Peabody Picture Vocabulary Scores; PRM, Pattern Recognition Memory; RAVLT, Rey Auditory Verbal Learning Test; RBANS, Repeatable Battery for the Assessment of Neuropsychological Status; RMF, Recognition Memory Faces; ROCFT, Rey-Osterreich Complex Figure Test; RT, reaction time ; RVP, Rapid Visual Information Processing ; SA-WAIS, South African-Wechsler Adult Intelligence Scale; SCWT, Stroop Colour-Word Test; S.D., standard deviation; SMTS \& DMTS, Simultaneous and Delayed Matching to Sample; SPM, Ravens's Standard Progressive Matrices; SRM, spatial recognition memory; SSP, spatial span; SWM, spatial working memory; SZ, schizophrenia; SZA, schizo-affective; TMT-A, Trail Making Test A; TMT-B, Trail Making Test B; VERT-K, Vienna Emotion Recognition Tasks; VIEMER-K, Vienna Memory of Emotion Recognition; VPA, Verbal Paired Associates; WAIS-R, Wechsler Adult Intelligence Scale - Revised; WASI, Wechsler Abbreviated Scale of Intelligence; WCST, Wisconsin Card Sorting Test; WM, working memory; WM-MA,

Working Memory-Mental Arithmetic Test; WMS, Wechsler Memory Scale; YMRS, Young Mania Rating Scale. 
correlates were more extensive in BD II than in BD I patients. The authors hypothesized that persistent depression, rather than mania, may represent a key pathophysiological factor or, alternatively, that BD II represents a clinical phenotype at risk for developing cognitive abnormalities. Although it is not possible to know whether the neuroanatomical substrate of cognitive function in patients deviates from that of normal subjects since the study did not include HCs, this does not invalidate the detection of differences between the two subgroups of BD patients. Moreover, the BD II sample was small $(n=11)$.

In conclusion, most studies failed to detect significant differences in the IQ of BD II patients compared with BD I patients or healthy subjects.

\section{Attention and psychomotor speed}

In the selected studies attention and psychomotor speed have been assessed by different tests [Trail Making Test (TMT), Digits Span, Digit Symbol, Stroop Test, simple reaction time, Continuous Performance Test (CPT), Coding Task in RBANS (Repeatable Battery for the Assessment of Neuropsychological Status), Rapid Visual Information Processing from the Cambridge Neuropsychological Test Automated Battery (CANTAB)]. In three studies euthymic BD II patients were found to have poorer performance compared with HCs (Torrent et al. 2006; Andersson et al. 2008; Dittmann et al. 2008), as well as BD I patients; there were no differences between these two groups (Torrent et al. 2006; Dittmann et al. 2008). In another more recent study BD II patients in an interepisodic phase had an intermediate performance between BD I and healthy subjects (Hsiao et al. 2009). In a study (Harkavy-Friedman et al. 2006) assessing BD patients with a depressive episode, BD II patients had a worse performance in the Digit Symbol subtest, and performed worse than BD I patients in the Stroop Test. However, no significant differences were found in the TMT Test A (TMT-A) and CPT; this is the only study that assessed sustained attention by means of the CPT. Holmes et al. (2008) detected deficits in sustained attention in depressed medicated BD patients, particularly those with BD II, but not unmedicated BD patients, and suggested that such attention impairment may be specifically related to treatment with mood-stabilizing agents, therefore representing a medication side-effect or being the combination of the two.

On the other hand, two studies did not find impaired attention using the Digits Forward (Savitz et al. 2008; Simonsen et al. 2008) and three of them did not find impairment in psychomotor speed using the TMT-A with respect to HC subjects
(Harkavy-Friedman et al. 2006; Hsiao et al. 2009) or with respect to normative data (Summers et al. 2006).

Hence, concerning this domain the results are contradictory, probably due in part to the attentional measures used. Most measures implicate other components such as working memory and psychomotor speed, which may be less sensitive. In this regard, only one of the studies used the CPT, a widespread measure of sustained attention.

\section{Learning and memory}

\section{Verbal memory}

Verbal memory is usually evaluated with tests including word lists and story recall.

In four out of nine studies, BD II patients scored significantly worse than control subjects or normative data in this domain (Martínez-Arán et al. 2004b; Summers et al. 2006; Torrent et al. 2006; Andersson et al. 2008) and two of them showed that the BD I group performed worse on some measures of verbal memory than the BD II group (Martínez-Arán et al. 2004a; Torrent et al. 2006). Of these reports, only the study conducted by Torrent et al. (2006) assessed exclusively euthymic patients. In the study by Summers et al. (2006) BD I patients were only impaired in verbal recognition memory, whereas BD II patients presented more impairment in verbal memory measures. The small sample size of the BD II group $(n=11)$ should, however, be taken into account.

In contrast, deficits in verbal memory in BD II patients were not found in five studies (HarkavyFriedman et al. 2006; Dittmann et al. 2008; Savitz et al. 2008; Simonsen et al. 2008; Hsiao et al. 2009), whereas in four of them a significantly worse performance in BD I patients was observed (Dittmann et al. 2008; Savitz et al. 2008; Simonsen et al. 2008; Hsiao et al. 2009). However, Harkavy-Friedman et al. (2006) did not find significant differences between BD I and BD II patients with a depressive episode and suicide attempts and HCs.

Simonsen et al. (2011) investigated whether neurocognitive dysfunction depends more on the history of psychosis or the diagnostic subtype and found that the latter only had significant main effect on two verbal recall measures, whereas history of psychosis influenced all subscores.

Discrepancies between studies do not allow us to draw conclusive results; nonetheless, four out of nine studies detected poorer performance in verbal memory in BD II patients. These discrepancies might be due to the different memory tasks used. Nevertheless, other factors could explain these discrepant findings such as the above-mentioned, history of psychoses or 
the presence of subclinical symptoms, among other factors that require further research.

\section{Visual memory}

Visual memory has been assessed in eight studies. In three of them BD II patients had a worse performance than healthy subjects (Andersson et al. 2008; Dittmann et al. 2008) or with respect to normative data (Summers et al. 2006). In the study by Dittmann et al. (2008), both BD I and BD II groups significantly differed from the HC group but the two patient groups did not differ from each other. However, the findings reported by Andersson et al. (2008) did not reflect important functional impairment since the difference did not exceed 0.5 s.D. below the normative mean.

In contrast, five studies did not detect impaired visual memory in BD II patients (Harkavy-Friedman et al. 2006; Taylor Tavares et al. 2007; Holmes et al. 2008; Savitz et al. 2008; Hsiao et al. 2009). Savitz et al. (2008) suggested that verbal and, perhaps, visual recall deficits distinguish BD I patients from individuals with bipolar spectrum disorders. However, in two of them, BD I patients also did not differ from the HC group in visual memory (Harkavy-Friedman et al. 2006; Hsiao et al. 2009).

Only three out of eight studies detected visual memory deficits in BD II patients. Therefore, the visual memory impairment, if confirmed, would be relatively small, and may depend on factors such as mild depressive symptoms or prior history of psychotic symptoms.

\section{Executive functions and working memory}

Working memory

Most of the studies reported that the working memory and executive function domain is impaired in BD II patients. In studies with samples formed of euthymic patients or patients with mild or residual symptomatology, deficits in some working memory measures were detected (Summers et al. 2006; Torrent et al. 2006; Andersson et al. 2008; Dittmann et al. 2008; Simonsen et al. 2008; Hsiao et al. 2009). In this regard, Summers et al. (2006) found that BD II patients were significantly more impaired than BD I patients. Moreover, in a sample of depressed BD I and BD II suicide attempters, a poorer working memory performance was observed in BD II in two measures while BD I patients presented poorer performance only in one measure when compared with HCs (Harkavy-Friedman et al. 2006).

On the other hand, two studies failed to find impaired working memory in depressed BD II patients (Taylor Tavares et al. 2007; Holmes et al. 2008) as well as a study assessing euthymic patients (Savitz et al. 2008).

In summary, six studies found impaired working memory while three of them failed to detect it; therefore, we can hypothesize that a working memory deficit may be one of the features of cognitive dysfunction associated with BD II.

\section{Verbal fluency}

Regarding phonemic verbal fluency, five out of six studies did not find a deficit in BD II patients (Summers et al. 2006; Torrent et al. 2006; Andersson et al. 2008; Savitz et al. 2008; Simonsen et al. 2008), while Harkavy-Friedman et al. (2006) pointed out that BD II patients had a worse performance than HC subjects in this task. It is also important to underline that BD II subjects in the latter study were depressed, so this finding may be related to findings reported by Martínez-Arán et al. (2002, 2004b) where the only significant difference between euthymic patients and depressed patients was observed in verbal fluency, suggesting that performance in the phonemic fluency task may be state-dependent.

Three studies examined semantic verbal fluency and two of them found that both BD I and BD II participants performed worse than HCs (Torrent et al. 2006; Simonsen et al. 2008). On the other hand, one study showed that only BD I patients performed worse than controls and BD II patients did not significantly differ from both BD I and HC groups (Dittmann et al. 2008).

\section{Cognitive flexibility}

The Wisconsin Card Sorting Test measures executive function, especially concept formation and cognitive flexibility. Three studies using this test did not find impaired BD II patients (Summers et al. 2006; Torrent et al. 2006; Savitz et al. 2008). However, Torrent et al. (2006) found that BD II patients, as well as BD I patients, showed a trend towards a higher number of perseverative errors compared with HCs, which may also be related to greater impulsivity. In two studies, cognitive flexibility was evaluated with another measure, the Intra-Dimensional/Extra-Dimensional Set-Shift subtest from CANTAB, with contradictory results; one of them found that unmedicated BD II depressed subjects demonstrated intact performance (Taylor Tavares et al. 2007) and the other found that BD II patients scored significantly lower than BD I patients (Summers et al. 2006).

The TMT Test B (TMT-B) is used as a cognitive flexibility measure as well as a working memory measure. Three studies out of five did not find differences between the $\mathrm{BD}$ II and $\mathrm{HC}$ groups 
(Harkavy-Friedman et al. 2006; Torrent et al. 2006; Hsiao et al. 2009). In the study by Summers et al. (2006) BD II patients were not impaired with respect to the normative data on the TMT-B, although they scored significantly lower than BD I participants on this measure. On the other hand, in the sample of Dittmann et al. (2008) BD II patients performed significantly poorer than the HC group in this measure, although BD I patients did not differ from both groups. However, in the study by Torrent et al. (2006), although no significant differences were found, a trend towards a poorer performance was detected in both BD subtypes when compared with HCs.

Overall, probably there is a decrease of cognitive flexibility in BD II.

\section{Inhibitory control}

The Stroop Colour and Word Test (SCWT) is a measure of selective attention, but the interference measure on the SCWT is usually considered a cognitive flexibility and, therefore, executive function measure. Three out of four studies found that BD II participants had a significantly poorer performance than the HC group (Torrent et al. 2006; Andersson et al. 2008; Simonsen et al. 2008). In one of them, both BD I and BD II groups did not differ significantly among each other (Simonsen et al. 2008) and in another the BD II patients showed an intermediate level of performance between the BD I and control group (Torrent et al. 2006). Another study, detected as well that the BD II group was impaired with respect to normative data regarding this measure and scored significantly lower than the BD I group as well (Summers et al. 2006).

In summary, all studies reported impaired inhibitory control as measured by the SCWT in BD II.

\section{Other neuropsychological domains}

\section{Motor functioning}

One study assessed motor functioning by the performance of two tasks (Harkavy-Friedman et al. 2006). In the Finger Tapping Test, no differences were detected between the BD I, BD II and HC groups, but depressed BD II patients performed significantly worse than BD I patients and HC subjects on a simple motor task.

Another study (Berns et al. 2002) failed to find differences in a reaction time task between euthymic BD II and HC subjects, but different brain responses when performing novel motor-spatial sequences were shown. The authors suggested that the adaptation to a novel sequence occurs by different mechanisms in patients, with a widespread medial prefrontal and limbic activation. However, the lack of a BD I group made it impossible to know whether both subtypes had a similar activation of brain areas.

Motor functioning is a neglected domain, so future studies should focus on it, also given the task simplicity and compatibility with concomitant brain imaging analysis.

\section{Visual/constructional abilities}

Only one study (Dittmann et al. 2008) evaluated this domain and found that BD II patients showed significant deficits in this cognitive function compared with HC subjects, while BD I patients did not differ from any of the groups.

\section{Affective processing}

In a study by Holmes et al. (2008), the medicated BD group exhibited greater response latency than unmedicated BD and healthy subjects and made more omission errors during the happy condition than in the sad condition in an affective processing task, indicating a potential attentional bias in subjects with bipolar depression on mood-stabilizing medications. The sample included both depressed BD I and BD II participants; however, additional tests were run after omitting BD I subjects to determine the impact of this smaller group without altering the results. The authors consider that affective blunting may occur as a result of treatment with mood-stabilizing medications. In another study (Summers et al. 2006), BD patients underperformed with respect to normative data in accuracy on the expression of surprise and there were no significant differences between the BD I and BD II groups. Patients with residual depression were less sensitive to expressions of happiness and anger than euthymic patients. They suggested that a poor emotion processing in these patients is due to depressionrelated cognitive deficits. On the other hand, Derntl et al. (2009) observed a reduced emotion recognition performance in BD I but not BD II patients. BD I participants tended to misinterpret especially sadness as fear. These results were not correlated with either residual symptoms or other various clinical characteristics.

Although these studies reported differences in the emotion to which the BD patients are biased in affective processing, they support the notion that BD patients are impaired in recognizing emotions, regardless of diagnostic subtype.

\section{Discussion}

There is a paucity of studies addressing cognitive deficits in BD II, even more so regarding patients in euthymia. The latter are more suitable in order to 
Table 2. Methodological issues of comparative studies on cognition: role of factors involved in cognition

- No consistent results about the effect of pharmacological treatment on cognition. There is a lack of control due to polypharmacy


this variable

- History of psychosis may partly account for the cognitive dysfunction even when controlling for bipolar diagnostic subtype ${ }^{\mathrm{b}, \mathrm{c}}$

- Childhood trauma has been reported to influence cognition negatively ${ }^{\mathrm{d}}$. It might be important to control for. Other factors to control for are factors related to prenatal development and obstetric complications ${ }^{\mathrm{e}}$, and factors associated with the neurodevelopmental process in general

- Potentially confounding variables in neuropsychological analyses are co-morbid alcohol abuse ${ }^{\mathrm{f}}$, attention deficit/hyperactivity disorderg,h,i and other co-morbid conditions

\footnotetext{
a Martínez-Arán et al. (2000). ${ }^{b}$ Martínez-Arán et al. (2008). ${ }^{c}$ Simonsen et al. (2011). d Savitz et al. (2008). e Martino et al. (2009) ${ }^{\mathrm{f}}$ Sánchez-Moreno et al. (2009). ${ }^{\mathrm{g}}$ Pavuluri et al. (2006). ${ }^{\mathrm{h}}$ Henin et al. (2007). ${ }^{\mathrm{i}}$ Rucklidge (2006).
}

evaluate the magnitude and the permanence of cognitive disturbance. Furthermore, methodological differences exist regarding comparative groups, such as heterogeneity of samples or inclusion of different mood states of the illness, for instance, making it difficult to draw conclusions. Some of the studies evaluated differences in neuropsychological performance between BD I and BD II; other studies compared depressed BD II patients with major depressive disorder; further studies focused on the comparison with other bipolar spectrum patients. Small sample-sized studies, especially regarding BD II patients, might have determined type I or type II errors, so larger samples should be analysed in order to reach firmer conclusions. Some studies were conducted in unmedicated patients, since treatments may play an important role in cognitive deficits, although available data regarding the effects of mood-stabilizers on cognition are inconsistent, possibly due to methodological issues (Goldberg \& Chengappa, 2009; Vieta, 2009; Balanzá-Martínez et al. 2010). The cognitive deficits observed in BD are not simply the effect of medication since these same cognitive deficits have been observed in unaffected relatives (Arts et al. 2008; Bora et al. 2009). There is also a lack of consensus on neuropsychological tasks that assess different cognitive functions and the classification of tasks included under each cognitive domain; the same cognitive task may be used as a measure of different cognitive domains in different studies, because the performance in most tests involves more than one cognitive process. Depending on the classification of the neuropsychological measures on the different neurocognitive domains, the results of meta-analyses or systematic reviews may vary. The literature has suggested an association between cognitive impairment and several clinical factors, such as residual affective symptoms, number and subtype of episodes, age at illness onset and number of admissions (Martínez-Arán et al. 2004a, b). Nevertheless, there are other important clinical factors related to worse cognitive functioning that have not been systematically investigated. Table 2 summarizes the methodological issues of comparative studies regarding the role of factors involved in cognition.

Regarding the selected studies, some of them conclude that there are no essential differences in neuropsychological profiles between BD I and BD II patients (Dittmann et al. 2008). In the above-mentioned study, BD I patients showed significantly lower scores in psychomotor speed, working memory, verbal learning, delayed memory and executive functions than HCs, while BD II patients showed significant deficits in psychomotor speed, working memory, visual/ constructional abilities and executive functions, but not on verbal learning and delayed memory. No significant differences among clinical groups were detected on any tested domain, supporting a similar pattern of cognitive deficits.

Other studies found that BD II patients had an intermediate level of performance between BD I and HC groups in verbal memory (Martínez-Arán et al. 2004b; Torrent et al. 2006) and executive functions (Torrent et al. 2006). Similarly, some authors suggest that BD I patients have more widespread cognitive dysfunction than BD II patients (Simonsen et al. 2008; Hsiao et al. 2009). In the study by Simonsen et al. (2008) BD II patients showed reduced performance on certain measures of attention and executive function (working memory, verbal fluency and interference control), while the BD I group showed reduced performance on all verbal memory measures and on most measures of attention and executive function (working memory, fluency, interference control and set-shifting). Moreover, a higher proportion of BD I patients had clinically significant cognitive impairment compared with BD II patients, and they concluded that both groups 
have different neurocognitive profiles. In the study by Hsiao et al. (2009), while BD II patients showed a reduction only in working memory and psychomotor speed, BD I patients also showed a reduction in verbal memory and executive function.

Nevertheless, two studies suggest that cognitive deficits are more severe and pervasive in BD II than BD I patients (Harkavy-Friedman et al. 2006; Summers et al. 2006). In the study by Summers et al. (2006), whereas the BD I group was only impaired in verbal recognition and spatial working memory, the BD II group was also impaired in these measures and additionally in other memory and executive measures. These authors point out that recurrent depressive episodes, rather than mania, may have a more detrimental and lasting effect on cognition. Regarding the study conducted by Harkavy-Friedman et al. (2006), their sample of BD II patients may represent a greater severity of illness than those with BD II who have never committed a suicide attempt; however, they suggested that BD II is a serious disorder with a distinguishing pattern of neuropsychological functioning.

Finally, two studies failed to find deficits in the BD II group. The first study found that unmedicated depressed BD II subjects displayed relatively intact cognitive function (Taylor Tavares et al. 2007). The latter found that BD II patients did not differ significantly from their unaffected relatives, whereas BD I patients showed impaired verbal memory (Savitz et al. 2008). However, the sample size of BD II patients in these two studies was relatively small.

Noteworthy, also, is the study by Andersson et al. (2008), where performance of BD II patients was significantly worse than that of HCs on all measures, except for phonemic verbal fluency. These authors discussed the functional significance of neuropsychological impairment and suggested that differences regarding some aspects of executive function may be related to psychomotor speed, and not primarily to dysexecutive functioning, taking into consideration their findings in electrophysiological index.

Therefore, the results of these studies are inconsistent, possibly due to different methodologies used. For example, a factor that may predispose to a greater cognitive dysfunction in BD II than BD I is the increased prevalence of subclinical depressive symptoms in BD II (Benazzi, 2001). Despite the scarcity of studies, the neuropsychological profiles of both diagnostic subtypes are different and, except for two studies, all of them detected cognitive deficits in BD II. The main findings include impaired working memory and some measures of executive functions (inhibitory control) and approximately half of the studies also detect verbal memory impairment.
Underlying mechanisms for differences in cognitive functioning between the two diagnostic subtypes could be due to either variations in frequency and severity of symptoms, as well as residual depressive symptoms, or to different genetic liability (Dittmann et al. 2008), or these may indicate neurobiological differences (Simonsen et al. 2008). These findings showing distinct cognitive profiles in both subtypes could lead to better identification of cognitive endophenotypes in BD. BD I would be nearer schizophrenia and BD II would show a lesser degree of cognitive impairment, reflecting the idea of a continuum in the psychoses (Torrent et al. 2006; MartínezArán et al. 2008).

\section{Future directions}

Future studies should routinely assess cognitive functioning in euthymic patients with both subtypes of BD. Furthermore, large samples are to be highly recommended. One implication arising from this review is that future neurocognitive research needs to focus on individuals with BD and concomitant history of psychosis separately from those without a history of psychosis in order to study the impact of this variable on neuropsychological performance. Similarly to the above-mentioned study by Simonsen et al. (2011), Martínez-Arán et al. (2008) also suggested that the history of psychosis may partly account for the cognitive dysfunction seen in euthymic patients, especially with regard to persistent verbal memory dysfunction as well as some executive dysfunctions. Future studies should take into account residual symptoms, which are not frequently measured, since these might possibly explain some persistent cognitive deficits (Martino et al. 2009); furthermore a consensual definition of subclinical symptoms is necessary (Daban et al. 2006). Other variables to consider should be the polarity of the last episode, predominant polarity and the period of time from the last recurrence. Sustained attention should be routinely assessed, since attention is a function that influences other cognitive domains. Motor functioning is another aspect to evaluate (Balanzá-Martínez et al. 2008). In a recent study it was suggested that motor speed may be a potential endophenocognitype for both schizophrenia and BD I patients (Salazar-Fraile et al. 2009). It is necessary to reach a consensus on the most appropriate cognitive tests for the assessment of cognitive impairment in BD, in order to obtain comparable studies and replicate findings.

Differentiation between BD I and BD II patients will be helpful in order to determine cognitive endophenotypes in BD. Findings on neurocognitive differences between the two subtypes can be used as 
markers in research for underlying neurobiological distinguishers using neuroimaging techniques. Neuroimaging and genetics might be useful in validating BD II diagnostic subtype (Vieta \& Suppes, 2008). Some genetic studies have suggested that BD II and BD I 'breed true' in families; therefore, in further studies it would be interesting to correlate clinical and neuropsychological data with genetic data in BD II patients. Although this specific issue has not been extensively examined in this paper, focused basically on cognition, it could be important to do so in a future report.

Differences in cognitive dysfunction between both subtypes may have an effect on psychosocial functioning, treatment adherence as well as the possibility to benefit from psychoeducational programmes. Neurocognitive rehabilitation should consider differences in cognitive profiles in order to design specific programmes aiming to treat prevailing cognitive dysfunctions for each subtype.

According to the conclusions of this systematic review, though still not clearly established, it appears that there are subtle differences between BD I and BD II regarding cognition; deficits in BD II subjects were identified mainly in the areas of working memory, inhibitory control and verbal memory as well. Nowadays, BD II is classified as a distinct category within mood disorders, but the definition and boundaries deserve further clarification (Vieta \& Suppes, 2008).

\section{Acknowledgements}

This review was supported by the Instituto de Salud Carlos III, Centro de Investigación en Red de Salud Mental, CIBERSAM and the Generalitat de Catalunya to the Bipolar Disorders Group (2009 SGR 1022) and the following grant support by the Spanish Ministry of Science and Innovation (CP07/00144, PI080180 and PI08/90094). C.M.B. is funded by the Spanish Ministry of Education through an FPU (Formación de Profesorado Universitario) scholarship.

\section{Declaration of Interest}

A.M.-A. has received research funding from the Spanish Ministry of Innovation. E.V. has received research grants and served as consultant, advisor or speaker for the following companies: Almirall, AstraZeneca, Bristol-Myers Squibb, Eli Lilly, Forest Research Institute, Geodon Richter, GlaxoSmithKline, Janssen-Cilag, Jazz, Lundbeck, Merck, Novartis, Organon, Otsuka, Pfizer Inc., Sanofi-Aventis, Servier, Solvay, Schering-Plough, Takeda, United Biosource Corporation, and Wyeth. E.V. also received research funding from the Spanish Ministry of Science and
Innovation, the Stanley Medical Research Institute and the 7th Framework Program of the European Union.

\section{References}

Akiskal HS (1996). The prevalent clinical spectrum of bipolar disorders: beyond DSM-IV. Journal of Clinical Psychopharmacology 16, 4S-14S.

Andersson S, Barder HE, Hellvin T, Lovdahl H, Malt UF (2008). Neuropsychological and electrophysiological indices of neurocognitive dysfunction in bipolar II disorder. Bipolar Disorders 10, 888-899.

Angst J (1998). The emerging epidemiology of hypomania and bipolar II disorder. Journal of Affective Disorders 50, 143-151.

APA (2000). Diagnostic and Statistical Manual of Mental Disorders, 4th edn, text revision (DSM-IV-TR). American Psychiatric Press: Arlington, VA.

Arts B, Jabben N, Krabbendam L, van Os J (2008). Meta-analyses of cognitive functioning in euthymic bipolar patients and their first-degree relatives. Psychological Medicine 38, 771-785.

Balanzá-Martínez V, Rubio C, Selva-Vera G, Martinez-Arán A, Sánchez-Moreno J, Salazar-Fraile J, Vieta E, Tabarés-Seisdedos R (2008). Neurocognitive endophenotypes (endophenocognitypes) from studies of relatives of bipolar disorder subjects: a systematic review. Neuroscience and Biobehavioral Reviews 32, 1426-1438.

Balanzá-Martínez V, Selva G, Martínez-Arán A, Prickaerts J, Salazar J, González-Pinto A, Vieta E, Tabarés-Seisdedos R (2010). Neurocognition in bipolar disorders - a closer look at comorbidities and medications. European Journal of Pharmacology 626, 87-96.

Benazzi F (1999). Prevalence of bipolar II disorder in atypical depression. European Archives of Psychiatry and Clinical Neuroscience 249, 62-65.

Benazzi F (2001). Prevalence and clinical correlates of residual depressive symptoms in bipolar II disorder. Psychotherapy and Psychosomatics 70, 232-238.

Berk M, Dodd S (2005). Bipolar II disorder: a review. Bipolar Disorders 7, 11-21.

Berns GS, Martin M, Proper SM (2002). Limbic hyperreactivity in bipolar II disorder. American Journal of Psychiatry 159, 304-306.

Bora E, Yucel M, Pantelis C (2009). Cognitive endophenotypes of bipolar disorder: a meta-analysis of neuropsychological deficits in euthymic patients and their first-degree relatives. Journal of Affective Disorders 113, 1-20.

Bruno SD, Papadopoulou K, Cercignani M, Cipolotti L, Ron MA (2006). Structural brain correlates of IQ changes in bipolar disorder. Psychological Medicine 36, 609-618.

Daban C, Martínez-Arán A, Torrent C, Tabarés-Seisdedos R, Balanzá-Martínez V, Salazar-Fraile J, Selva-Vera G, Vieta E (2006). Specificity of cognitive deficits in bipolar disorder versus schizophrenia. A systematic review. Psychotherapy and Psychosomatics 75, 72-84. 
Derntl B, Seidel EM, Kryspin-Exner I, Hasmann A, Dobmeier M (2009). Facial emotion recognition in patients with bipolar I and bipolar II disorder. British Journal of Clinical Psychology 48, 363-375.

Dittmann S, Hennig-Fast K, Gerber S, Seemuller F, Riedel M, Emanuel SW, Langosch J, Engel RR, Moller HJ, Grunze HC (2008). Cognitive functioning in euthymic bipolar I and bipolar II patients. Bipolar Disorders 10, 877-887.

Engstrom C, Brandstrom S, Sigvardsson S, Cloninger R, Nylander PO (2003). Bipolar disorder. II: personality and age of onset. Bipolar Disorders 5, 340-348.

Glahn DC, Bearden CE, Niendam TA, Escamilla MA (2004). The feasibility of neuropsychological endophenotypes in the search for genes associated with bipolar affective disorder. Bipolar Disorders 6, 171-182.

Goldberg JF, Chengappa KN (2009). Identifying and treating cognitive impairment in bipolar disorder. Bipolar Disorders 11 (Suppl. 2), 123-137.

Harkavy-Friedman JM, Keilp JG, Grunebaum MF, Sher L, Printz D, Burke AK, Mann JJ, Oquendo M (2006). Are BPI and BPII suicide attempters distinct neuropsychologically? Journal of Affective Disorders 94, 255-259.

Henin A, Mick E, Biederman J, Fried R, Wozniak J, Faraone SV, Harrington K, Davis S, Doyle AE (2007). Can bipolar disorder-specific neuropsychological impairments in children be identified? Journal of Consulting and Clinical Psychology 75, 210-20.

Holmes MK, Erickson K, Luckenbaugh DA, Drevets WC, Bain EE, Cannon DM, Snow J, Sahakian BJ, Manji HK, Zarate Jr. CA (2008). A comparison of cognitive functioning in medicated and unmedicated subjects with bipolar depression. Bipolar Disorders 10, 806-815.

Hsiao YL, Wu YS, Wu JY, Hsu MH, Chen HC, Lee SY, Lee IH, Yeh TL, Yang YK, Ko HC, Lu RB (2009). Neuropsychological functions in patients with bipolar I and bipolar II disorder. Bipolar Disorders 11, 547-554.

Kapczinski F, Vieta E, Andreazza AC, Frey BN, Gomes FA, Tramontina J, Kauer-Sant'anna M, Grassi-Oliveira R, Post RM (2008). Allostatic load in bipolar disorder: implications for pathophysiology and treatment. Neuroscience and Biobehavioral Reviews 32, 675-692.

Martínez-Arán A, Penades R, Vieta E, Colom F, Reinares M, Benabarre A, Salamero M, Gasto C (2002). Executive function in patients with remitted bipolar disorder and schizophrenia and its relationship with functional outcome. Psychotherapy and Psychosomatics 71, 39-46.

Martínez-Arán A, Torrent C, Tabarés-Seisdedos R, Salamero M, Daban C, Balanzá-Martínez V, Sánchez-Moreno J, Manuel GJ, Benabarre A, Colom F, Vieta E (2008). Neurocognitive impairment in bipolar patients with and without history of psychosis. Journal of Clinical Psychiatry 69, 233-239.

Martínez-Arán A, Vieta E, Colom F, Reinares M, Benabarre A, Gastó C, Salamero M (2000). Cognitive dysfunctions in bipolar disorder: evidence of neuropsychological disturbances. Psychotherapy and Psychosomatics 69, 2-18.

Martínez-Arán A, Vieta E, Colom F, Torrent C, Sánchez-Moreno J, Reinares M, Benabarre A,
Goikolea JM, Brugue E, Daban C, Salamero M (2004a). Cognitive impairment in euthymic bipolar patients: implications for clinical and functional outcome. Bipolar Disorders 6, 224-232.

Martínez-Arán A, Vieta E, Reinares M, Colom F, Torrent C, Sánchez-Moreno J, Benabarre A, Goikolea JM, Comes M, Salamero M (2004b). Cognitive function across manic or hypomanic, depressed, and euthymic states in bipolar disorder. American Journal of Psychiatry 161, 262-270.

Martino DJ, Marengo E, Igoa A, Scapola M, Ais ED, Perinot L, Strejilevich SA (2009). Neurocognitive and symptomatic predictors of functional outcome in bipolar disorders: a prospective 1 year follow-up study. Journal of Affective Disorders 116, 37-42.

Pallanti S, Quercioli L, Pazzagli A, Rossi A, Dell'Osso L, Pini S, Cassano GB (1999). Awareness of illness and subjective experience of cognitive complaints in patients with bipolar I and bipolar II disorder. American Journal of Psychiatry 156, 1094-1096.

Pavuluri MN, Schenkel LS, Aryal S, Harral EM, Hill SK, Herbener ES, Sweeney JA (2006). Neurocognitive function in unmedicated manic and medicated euthymic pediatric bipolar patients. American Journal of Psychiatry 163, 286-293.

Robinson LJ, Thompson JM, Gallagher P, Goswami U, Young AH, Ferrier IN, Moore PB (2006). A meta-analysis of cognitive deficits in euthymic patients with bipolar disorder. Journal of Affective Disorders 93, 105-115.

Rucklidge JJ (2006). Impact of ADHD on the neurocognitive functioning of adolescents with bipolar disorder. Biological Psychiatry 60, 921-928.

Salazar-Fraile J, Balanzá-Martínez V, Selva-Vera G, Martínez-Arán A, Sánchez-Moreno J, Vieta E, Gómez-Beneyto M, Tabarés-Seisdedos R (2009). Motor speed predicts stability of cognitive deficits in both schizophrenic and bipolar I patients at one-year follow-up. European Journal of Psychiatry 23, 184-197.

Sanchez-Moreno J, Martinez-Aran A, Colom F, Scott J, Tabares-Seisdedos R, Sugranyes G, Torrent C, Daban C, Benabarre A, Goikolea JM, Franco C, González-Pinto A, Ayuso-Mateos JL, Vieta E (2009). Neurocognitive dysfunctions in euthymic bipolar patients with and without prior history of alcohol use. Journal of Clinical Psychiatry 70, 1120-1127.

Savitz JB, Solms M, Ramesar RS (2005). Neurocognitive function as an endophenotype for genetic studies of bipolar affective disorder. NeuroMolecular Medicine 7, 275-286.

Savitz JB, van der Merwe L, Stein DJ, Solms M, Ramesar RS (2008). Neuropsychological task performance in bipolar spectrum illness: genetics, alcohol abuse, medication and childhood trauma. Bipolar Disorders 10, 479-494.

Simonsen C, Sundet K, Vaskinn A, Birkenaes AB, Engh JA, Færden A, Jónsdóttir H, Ringen PA, Opjordsmoen S, Melle I, Friis S, Andreassen OA (2011). Neurocognitive dysfunction in bipolar and schizophrenia spectrum disorders depends on history of psychosis rather than diagnostic group. Schizophrenia Bulletin 37, 73-83.

Simonsen C, Sundet K, Vaskinn A, Birkenaes AB, Engh JA, Hansen CF, Jónsdóttir H, Ringen PA, Opjordsmoen S, 
Friis S, Andreassen OA (2008). Neurocognitive profiles in bipolar I and bipolar II disorder: differences in pattern and magnitude of dysfunction. Bipolar Disorders 10, 245-255.

Summers M, Papadopoulou K, Bruno S, Cipolotti L, Ron MA (2006). Bipolar I and bipolar II disorder: cognition and emotion processing. Psychological Medicine 1799-1809.

Taylor Tavares JV, Clark L, Cannon DM, Erickson K, Drevets WC, Sahakian BJ (2007). Distinct profiles of neurocognitive function in unmedicated unipolar depression and bipolar II depression. Biological Psychiatry 62, 917-924.

Torrent C, Martínez-Arán A, Daban C, Sánchez-Moreno J, Comes M, Goikolea JM, Salamero M, Vieta E (2006).

Cognitive impairment in bipolar II disorder. British Journal of Psychiatry 189, 254-259.

Torres IJ, Boudreau VG, Yatham LN (2007).

Neuropsychological functioning in euthymic bipolar disorder: a meta-analysis. Acta Psychiatrica Scandinavica 116 (Suppl. 434), 17-26.

Vieta E (2009). The influence of medications on neurocognition in bipolar disorder. Acta Psychiatrica Scandinavica 120, 414-415.

Vieta E, Colom F, Martínez-Arán A, Benabarre A, Gasto C (1999). Personality disorders in bipolar II patients. Journal of Nervous and Mental Disease 187, 245-248.

Vieta E, Gasto C, Otero A, Nieto E, Vallejo J (1997). Differential features between bipolar I and bipolar II disorder. Comprehensive Psychiatry 38, 98-101.

Vieta E, Suppes T (2008). Bipolar II disorder: arguments for and against a distinct diagnostic entity. Bipolar Disorders 10 163-178.

Warrington EK (1996). The Camden Memory Tests. Psychology Press: Hove, East Sussex.

Zarate Jr. CA, Tohen M, Land M, Cavanagh S (2000). Functional impairment and cognition in bipolar disorder. Psychiatric Quarterly 71, 309-329. 